\title{
Ceftazidime/Avibactam: Who Says You Can't Teach an Old Drug New Tricks?
}

\author{
Katie E. Barber ${ }^{1}$, Jessica K. Ortwine², Ronda L. Akins ${ }^{3}$
}

1. Department of Pharmacy Practice, University of Mississippi School of Pharmacy, Jackson, Mississippi, USA.

2. Department of Pharmacy, Parkland Hospital and Health System, Dallas, Texas, USA; University of Texas Southwestern Medical School, Dallas, Texas, USA. ${ }^{3}$ Department of Pharmacy, Methodist Charlton Medical Center, Dallas, Texas, USA; Department of Biological Sciences, University of Texas at Dallas, Richardson, Texas, USA

Received, October 14, 2016; Accepted, October 26, 2016; Published, November 2, 2016.

\begin{abstract}
Purpose: Gram-negative resistance continues to rise with treatment options becoming more limited. Ceftazidime/avibactam was recently approved in the United States and Europe, which combines an established third-generation cephalosporin with a new, unique, non- $\beta$-lactam $\beta$-lactamase inhibitor. This review conducts a thorough examination of structure, pharmacology, spectrum of activity, pharmacokinetics/pharmacodynamics, in vitro and clinical efficacy and safety/tolerability of ceftazidime/avibactam, as well as detailed future directions for the agent. Methods: Pubmed and clinicaltrials.gov searches, as well as abstracts from the 2015 Interscience Conference on Antimicrobial Agents and Chemotherapy/International Society of Chemotherapy (ICAAC/ICC) and ID Week meetings and the 2016 American Society of Microbiology Microbe meeting, were conducted from January 2004 - September 2016. Relevant search terms included ceftazidime, ceftazidime/avibactam, avibactam, NXL104 and AVE1330A. The US package insert for ceftazidime/avibactam (02/2015) and European public assessment report (06/2016) were also reviewed. Results: In vitro susceptibility for ceftazidime/avibactam displayed potent activity against many Enterobacteriaceae including extended-spectrum- $\beta$-lactamase (ESBL) and carbapenemase-producing strains, as well as Pseudomonas aeruginosa. Phase II clinical trials utilized for approval demonstrated comparable safety and efficacy to imipenem/cilistatin for treatment of complicated urinary tract infections $(70.4 \% \mathrm{vs} .71 .4 \%)$ and combined with metronidazole compared to meropenem in complicated intra-abdominal infections $(91.2 \%$ vs 93.4\%). Phase III data displayed non-inferior efficacy of ceftazidime/avibactam compared to doripenem for complicated urinary tract infections $(70.2 \%$ vs $66.2 \%)$ and combined with metronidazole compared to meropenem in complicated intra-abdominal infections $(82.5 \%$ vs $84.9 \%)$, as well as comparable safety. Ceftazidime/avibactam was well-tolerated but does require renal adjustments. Additionally, 3 case series and a single case report have demonstrated the potential for ceftazidime/avibactam against multidrug resistant organisms for compassionate use or failure after previous therapy. Conclusion: By adding avibactam to ceftazidime, clinicians' antimicrobial armamentarium is expanded, potentially increasing the ability to combat multi-drug resistant gram-negative pathogens, particularly ESBL and carbapenemase-producing organisms, as well as Pseudomonas aeruginosa.
\end{abstract}

This article is open to POST-PUBLICATION REVIEW. Registered readers (see "For Readers") may comment by clicking on ABSTRACT on the issue's contents page.

\section{INTRODUCTION}

Ceftazidime/avibactam is the first approved combination antibiotic to utilize the unique betalactamase inhibitor avibactam. This agent has demonstrated potent activity against numerous gram-negative organisms. New agents are a welcome addition to the antibiotic arsenal as these organisms continue to plague clinicians with unrelenting development of resistance, particularly noted in Enterobacteriaceae with the spread of extended-spectrum $\beta$-lactamases (ESBL) and carbapenem-resistant Enterobacteriaceae (CRE), as well as Acinetobacter baumannii and Pseudomonas aeruginosa.

Corresponding Author: Ronda L. Akins, Pharm.D. Department of Pharmacy; Methodist Charlton Medical Center; 3500 W. Wheatland Road; Dallas, Texas, USA; Email: RondaAkins@mhd.com 
While there are several reviews already published on this agent; this is the first review article to include recently published data from Phase III clinical studies as well as Phase II data, which was utilized for the initial approval of the agent. Furthermore, approval by the European Commission is also discussed, which includes an indication for nosocomial pneumonia. This particular indication has not been noted in any prior review.

In the past, the mainstay of therapy for these problematic pathogens has primarily been $\beta$-lactams; however, these organisms often possess enzymes rendering the preferred treatment ineffective. As a result, clinicians have resorted to using more toxic antibiotics such as the polymixins (colistimethate, polymixin B). However, newer agents including ceftazidime/avibactam, may provide a more efficacious and safer alternative in the treatment of these multi-drug resistant organisms. This is evident in the initial clinical trials (phase II and III) and postmarketing data, demonstrating that the ceftazidime/avibactam combination remained active against ceftazidime-resistant strains, as well as ESBL and CRE strains. Additionally, the agent was well tolerated with a similar safety profile as ceftazidime alone.

\section{HISTORICAL BACKGROUND}

Ceftazidime has a 30-year history which parallels the evolution of $\beta$-lactamases and as usage increased, numerous resistance mechanisms began limiting its utility (1-3). Originally approved in 1985, it has been utilized for numerous indications and in patient populations where MDR bacterial resistance is a primary concern, such as cystic fibrosis. However, as seen with other $\beta$-lactams, resistance ultimately emerged in Enterobacteriaceae and Pseudomonas aeruginosa.

Avibactam was designed in the mid-1990s, originally by Hoechst Marion Roussel, now marketed by Allergan (4). It is unique in the fact that it is a diazabicyclooctane (DBO), a class that mimics $\beta$-lactam; therefore a non- $\beta$-lactam $\beta$-lactamase inhibitor without any antimicrobial activity. The primary difficulty in development has been the DBO chemistry which requires multiple steps resulting in slow advancement. Early agents studied were weak inhibitors of class $A$ and $C \beta$-lactamases but the approved avibactam displays potent inhibition of both of these classes, with weaker inhibition against class D and no inhibition of metallo $\beta$-lactamases.

As a result of the continued rise in gram-negative bacterial resistance, the United States Food and Drug Administration (FDA) initiated the Generating Antibiotic Incentives Now (GAIN) Act, approved in 2012, which gives drug manufacturers five additional years of market exclusivity if the drug developed is designated as a "qualified infectious disease product (QIDP)" to promote development of novel agents (5). Ceftazidime/avibactam (Avycaz ${ }^{\mathbb{R}}$ ) was designated as a fast-track agent under QIDP criteria and approved by the US FDA in February 2015 based on phase II studies for use in complicated urinary tract and complicated intra-abdominal infections. Since then, subsequent phase III and pediatric studies have also been conducted. Additionally, in June 2016, the European Commission approved the combination agent under the trade name, Zavicefta for the treatment of complicated urinary tract and intra-abdominal infections, hospital-acquired pneumonia and infections due to gram-negative organisms with limited alternative treatment options. This review will examine all currently available data, through September 30, 2016, for ceftazidime/avibactam providing information relevant to the potential usage of this much needed agent for MDR gram-negatives.

\section{CHEMISTRY AND MECHANISM OF ACTION}

\subsection{Ceftazidime}

The basic structure of cephalosporins consists of a $\beta$ lactam ring fused with a six-member dihydrothiazine ring, with substitutions at $\mathrm{C} 3$ and $\mathrm{C} 7$. Unlike other third-generation cephalosporins, ceftazidime has a positively charged pyridine side chain at position 3, granting greater anti-pseudomonal activity. This also produces a zwitterion allowing for enhanced gramnegative cell membrane penetration and increased water solubility (6). The carboxypropyl group found on the aminothiazolyloximino side chain at position 7 also confers additional stability against $\beta$ lactamases produced by Enterobacteriaceae and $P$. aeruginosa (7). Ceftazidime exerts its activity by binding to penicillin-binding proteins (PBPs) within the cell wall, primarily PBP-3, interfering with cell division and leading to cell death (8). 

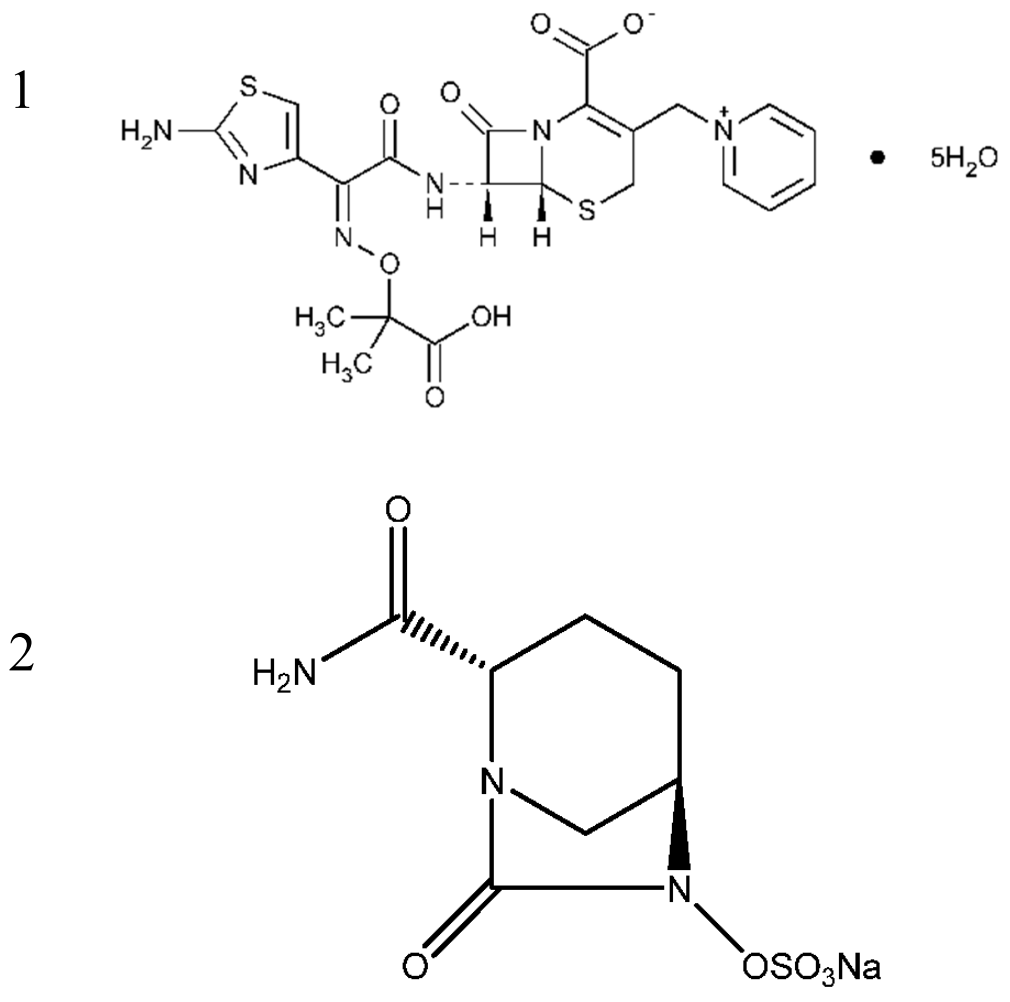

Figure 1. Ceftazidime (1) and avibactam (2) chemical structures

\subsection{Avibactam}

Avibactam is a new chemical entity, a non- $\beta$-lactam $\beta$-lactamase inhibitor, with no direct antibacterial activity. Structurally, it differs from other $\beta$ lactamase inhibitors lacking the distinctive 4membered $\beta$-lactam ring, instead containing a 5membered cyclic urea (8). Mechanistically, avibactam acts similarly to other $\beta$-lactamase inhibitors by creating a covalent bond at the activesite serine. The difference, however, is traditional $\beta$ lactamase inhibitors serve as enzyme substrates and irreversibly bind during the acylation step of enzyme inhibition then subsequently are either hydrolyzed or chemically rearranged allowing for partial or full return of enzyme activity after a period of time. Conversely, $\beta$-lactamase inhibition is a reversible process for avibactam due to structural uniqueness resulting in enzyme inhibition, followed by slow deacylation and ring closure leading to regeneration of active $\beta$-lactamase inhibitor. Ability to revert to the ring structure is likely due to less intrinsic strain on the 5-membered avibactam ring compared to the 4 -membered ring of traditional $\beta$-lactamase inhibitors.

\section{ANTIMICROBIAL SPECTRUM}

Ceftazidime on its own is still labile to AmpC $\beta$ lactamases commonly produced by $P$. aeruginosa as well as other higher level $\beta$-lactamases. Addition of avibactam reverses ceftazidime resistance and lowers the MIC of many gram-negative isolates to within susceptible range $(\leq 8 \mathrm{mg} / \mathrm{L})$ for Pseudomonas aeruginosa and Enterobacteriaceae (9). In vitro MIC testing of over 20,000 Enterobacteriaceae, including ESBL, KPC and AmpC-producing isolates, demonstrated a 5 -fold decrease in overall $\mathrm{MIC}_{90}$ (from 8 to $0.25 \mathrm{mg} / \mathrm{L}$ ) in activity of ceftazidime once avibactam was added (10). The MIC for all but 4 ESBL strains dropped to within the susceptible range upon avibactam exposure and a similar drop was seen for $97.5 \%$ of KPC isolates. Organisms known to potentially harbor AmpC enzymes (Enterobacter cloacae and Serratia marcescens) also demonstrated nearly $\quad 100 \% \quad$ susceptibility to ceftazidime/avibactam. Ceftazidime/avibactam has also shown promising results against class D OXA48 carbapenemases (11).

In addition, ceftazidime/avibactam improved coverage of $P$. aeruginosa compared to most other anti-pseudomonal agents. In vitro susceptibility to 
this combination demonstrated rates around 97\% when tested against 3,900 P. aeruginosa isolates, followed by amikacin at $94 \%$ (12). Susceptibility to all other $\beta$-lactams tested was less impressive with a range of 79 to $84 \%$. The greatest difference was demonstrated when testing ceftazidime/avibactam coverage against MDR $P$. aeruginosa. Of all agents tested, only 3 retained susceptibility rates above $25 \%$ - ceftazidime/avibactam (81\%), amikacin (88\%) and colistin (99\%).

While availability of ceftazidime/avibactam significantly helps to combat rising rates of MDR bacteria, there are important gaps in its antibacterial spectrum of activity. The combination remains essentially inactive against class $B$ metallo- $\beta$ lactamase-producing gram-negative organisms as well as most Acinetobacter species $(11,13)$. In addition, the combination does not have reliable coverage against anaerobic bacteria nor expands the limited gram-positive activity against streptococci of ceftazidime alone $(14,15)$.

Data from the European Centre for Disease Prevention and Control has documented that resistance to fluoroquinolones, third-generation cephalosporins, and aminoglycosides has dramatically increased in both E. coli and $K$. pneumoniae over the past four years $(16,17)$. Additionally, resistance to carbapenems, specifically in pseudomonal strains, has increased. With $\mathrm{MIC}_{50 / 90}$ values against Enterobacteriaeceae and $P$. aeruginosa of $0.12 / 0.25$ and $2 / 4 \mathrm{mg} / \mathrm{L}$, respectively, ceftazidime/avibactam represents a potential therapeutic option against these organisms (18).

\section{RESISTANCE}

The mechanisms of resistance with ceftazidime/avibactam are not unique to this agent. As previously discussed, most organisms producing class A $\beta$-lactamases (ESBL and KPC) remain highly susceptible. Unfortunately, this is not an absolute. In fact, Livermore et al., recently created ceftazidime/avibactam resistant KPC-3 isolates of Enterobacter cloacae and Klebsiella pneumoniae using a stepwise selection process leading to mutant selection at a $10^{-9}$ frequency (19). Interestingly, MIC decreases were observed among carbapenems and cephalosporins, except ceftazidime, during this investigation. Unfortunately, this resistance pattern is not isolated to laboratory observations. The first instance of a ceftazidime/avibactam resistant KPC-3 Klebsiella pneumoniae clinical isolate has been reported in a patient with no prior drug exposure
(20). Further exploration of KPC subtypes shows that the MIC of ceftazidime/avibactam against KPC3 is significantly higher than against KPC-2 variants (21).

Among class $C \beta$-lactamase-producers, a similar stepwise selection procedure was carried out using $P$. aeruginosa strains carrying derepressed AmpC genes (22). Results revealed low rates of spontaneous resistance with increased susceptibility to carbapenems, piperacillin-tazobactam and aztreonam among isolated mutants. While AmpC induction occurs more frequently with $2^{\text {nd }}$ and $3^{\text {rd }}$ generation cephalosporins, including ceftazidime, avibactam has shown greater activity over tazobactam or sulbactam (11). In vitro studies have demonstrated effective inhibition of AmpC in $E$. cloacae, a common pathogen with resistance development through this mechanism, with avibactam $(23,24)$. Therefore, by combining avibactam with ceftazidime, additional in vivo activity may be provided against $\mathrm{AmpC}$ induction in producing strains. However, no data is available regarding clinical outcomes in patients with infections caused by E. cloacae or other potential AmpC-producing organisms, so the true in vivo efficacy of this combination remains an area for further research.

In contrast to class $\mathrm{A}$ and $\mathrm{C} \beta$-lactamase resistance, which remains infrequent, organisms producing class B metallo- $\beta$-lactamases and most Acinetobacter baumanii harboring class D OXA- $\beta$ lactamases commonly display ceftazidime/avibactam resistance due to inability of avibactam to effectively inhibit these enzymes (25).

Additionally, by virtue of being a $\beta$-lactamase inhibitor, avibactam is expected only to protect ceftazidime against activity of $\beta$-lactamases. Resistance among Enterobacteriaceae is commonly due to the presence of $\beta$-lactamases, which is likely the reason susceptibility rates are affected so strongly in the presence of avibactam. However, $P$. aeruginosa is notorious for multiple mechanisms of resistance including porin and efflux pump mutations in addition to $\beta$-lactamase production (26). In order to investigate the role of these alternate mechanisms of $P$. aeruginosa resistance to ceftazidime/avibactam, Winkler et al. collected 10 historical $P$. aeruginosa isolates with no prior exposure to ceftazidime/avibactam but displayed resistance to this agent, then performed a series of experiments to determine cause for resistance (27). Their study found that $\beta$-lactamase enzymes, degree 
of $\beta$-lactamase expression and PBP target expression did not affect ceftazidime/avibactam susceptibility, and decreased porin expression possibly played only a minor role. The majority of ceftazidime/avibactam resistance was attributed to mutations causing upregulation of efflux pumps leading to decreased ceftazidime/avibactam exposure. Therefore, while this drug is effective against many $\beta$-lactamases, potential for cell membrane porin and efflux pump mutations to affect drug activity remain a concern.

\section{PHARMACOKINETICS/PHARMACODY NAMICS \\ 6.1.Ceftazidime}

Similar to other cephalosporins, ceftazidime pharmacokinetics have been well described in multiple patient populations including renal impairment, cystic fibrosis, critically ill and burn patients. Glomerular filtration is almost the exclusive route of elimination, thus clearance and half-life are significantly prolonged for patients with renal dysfunction. As a result, dosage adjustments are required in renal impairment.

\subsection{Avibactam}

In initial phase I studies evaluating pharmacokinetics of avibactam alone, avibactam was administered to healthy volunteers at doses ranging from $50-2000 \mathrm{mg}$ $(28,29)$. Total clearance was similar for all groups $(9.82-10.34 \mathrm{~L} / \mathrm{hr})$ except elderly women being slightly lower at $7.98 \mathrm{~L} / \mathrm{hr}$. Overall, avibactam was well tolerated with no significant differences in pharmacokinetics between gender or age. Linear pharmacokinetics of avibactam were observed which were not impacted by the addition of ceftazidime.

\subsection{Ceftazidime/avibactam}

Additional data from 2 phase I pharmacokinetic studies in healthy adults evaluated avibactam alone or combined with ceftazidime in a 1:4 (500/2000mg) ratio as single or multiple doses of ceftazidime/avibactam every 8 hours (Table 1) $(28,30)$. Avibactam peak plasma concentrations increased proportionally with ascending dosages, with minimal evidence of accumulation observed (28). Steady-state volume of distribution for avibactam was comparable (22L) to average adults (19L) suggesting adequate distribution to extravascular sites of infection.

The approved dosage of $500 \mathrm{mg}$ of avibactam with $2000 \mathrm{mg}$ of ceftazidime had a total clearance of 11.9 and $13.93 \mathrm{~L} / \mathrm{h}$, respectively, and a terminal halflife ranging from 1.5-2.7 hours over a 7-10 day course. Further demonstrating the renal route of elimination, combined with ceftazidime, $86.5 \%$ of avibactam was recovered from urine as unchanged drug; therefore undergoing minimal, if any, metabolism.

Effects of age and gender on avibactam pharmacokinetics and safety were evaluated in a phase I study with no differences between gender and only minimal changes with age (young vs elderly cohorts) (29).

Table 1. Avibactam Plasma Pharmacokinetic Parameters in Healthy Adult Participants after 500mg Dose Administered as a 2-Hour IV Infusion Every 8 Hours

\begin{tabular}{lllllll}
\hline Agent & $\mathrm{n}$ & $\mathrm{C}_{\max }(\mathrm{mg} / \mathrm{L})$ & AUC $(\mathrm{mg} \mathrm{h} / \mathrm{L})$ & Clearance $(\mathrm{L} / \mathrm{h})$ & $\mathrm{V}_{\mathrm{ss}}(\mathrm{L})$ & $t_{1 / 2}$ (hours) \\
\hline Day 1 & & & & & \\
A & $6^{\mathrm{a}}$ & 15.1 & 43.1 & 11.6 & & 1.4 \\
$\mathrm{C}+\mathrm{A}$ & $7^{\mathrm{a}}$ & 15.9 & 45.5 & 11 & 1.4 \\
A & $8^{\mathrm{b}}$ & 26.15 & 37.13 & 13.39 & 22.72 & 1.83 \\
$\mathrm{C}+\mathrm{A}$ & $8^{\mathrm{b}}$ & 23.33 & 37.15 & 13.36 & 25.43 & 2.16 \\
Day 7 & & & & & & 1.4 \\
A & $6^{\mathrm{a}}$ & 14.8 & 41.5 & 12.1 & & 1.4 \\
C+A & $6^{\mathrm{a}}$ & 15.0 & 42.2 & 11.9 & & \\
Day 10 & & & & & & \\
C+A & $8^{\mathrm{b}}$ & 22.11 & 35.89 & 13.93 & 24.22 & 1.5 \\
\hline
\end{tabular}

Abbreviations: AUC, area under the plasma concentration-time curve; A, avibactam; C+A, avibactam with 2000mg ceftazidime; $n$, number of participants; $\mathrm{C}_{\max }$, maximum plasma concentration; $\mathrm{V}_{\mathrm{ss}}$, volume of distribution at steady state; $t_{1 / 2}$, elimination half-life.

${ }^{a}$ Tominaga et al (30)

bMerdjan et al. (31) 
However, with only 33 patients included in this study, generalizability remains challenging. Comparable to other $\beta$-lactam/ $\beta$-lactamase inhibitors, as renal function declines clearance of avibactam is diminished warranting dose reductions when creatinine clearance is $\leq 50 \mathrm{~mL} / \mathrm{min}(31)$.

A phase I open label, single dose ceftazidime/avibactam evaluation was performed in pediatrics (32). Patients were divided into 4 age cohorts: cohort 1 ( $\geq 12-18$ years), cohort $2(\geq 6-<12$ years), cohort $3(\geq 2-<6$ years) and cohort 4 (3 months- $<2$ years). Thirty-two patients, 8 per cohort, were included; with cohort 1 and patients weighing $\geq 40 \mathrm{~kg}$ in cohort 2 receiving a dose of ceftazidime/avibactam 2000/500mg and remaining cohort 2 patients plus cohorts 3 and 4 receiving a dose of $50 / 12.5 \mathrm{mg} / \mathrm{kg}$. All dosages were administered over 2 hours. In cohorts 1-4, avibactam peak $(\mathrm{mg} / \mathrm{L})$ values were $15.1,14.1,13.7$ and 16.3 , with AUC (mg.hr/L) values of 36.4, 34.8, 43.3 and 49.0), respectively. These concentrations were comparable to adult peaks and AUCs from studies previously discussed. Not only are ceftazidime/avibactam pediatric pharmacokinetics similar to adults but the safety profile is comparative with no patients experiencing a severe adverse event.

\subsection{Pharmacodynamics}

It is well-described that ceftazidime displays timedependent pharmacodynamic activity requiring approximately $60 \%$ time above MIC for efficacy $(33,34)$. However, the question of avibactam dosing frequency needed further elucidation. To determine the most efficacious dosage, avibactam was evaluated as a continuous infusion at various concentrations $(0.5-10 \mathrm{mg} / \mathrm{L})$ in combination with ceftazidime or ceftaroline against $3 \beta$-lactamaseproducing Enterobacteriaceae (35). Avibactam concentrations of $\geq 2 \mathrm{mg} / \mathrm{L}$ provided activity against Enterobacteriaceae with CTX-M or AmpC enzymes. However, concentrations of $\geq 4 \mathrm{mg} / \mathrm{L}$ were required for adequate activity against KPC-producing $K$. pneumoniae. Dosages of avibactam at $500 \mathrm{mg}$ every 8 hours or $600 \mathrm{mg}$ every 12 hours achieved adequate concentrations for efficacy $(\geq 4 \mathrm{mg} / \mathrm{L})$ against all strains.

Similarly, Bowker and colleagues evaluated avibactam continuous infusions at various concentrations $\quad(2-10 \mathrm{mg} / \mathrm{L}) \quad$ combined with ceftazidime $2000 \mathrm{mg}$ or ceftaroline $600 \mathrm{mg}$ every 8 hours in an in vitro model over 48 hours against an AmpC-producing E. cloacae (36). At 24 hours, avibactam concentrations of $\geq 2 \mathrm{mg} / \mathrm{L}$ were capable of producing an $80 \%$ maximal effect. However, to sustain this effect at 48 hours, concentrations of 4$6 \mathrm{mg} / \mathrm{L}$ were required.

While continuous infusions of avibactam were utilized in these studies, other studies demonstrated intermittent infusions of $500 \mathrm{mg}$ avibactam every 8 hours produced concentrations $\geq 4 \mathrm{mg} / \mathrm{L}$ for majority of the dosing interval (9). Therefore, the approved dosage should achieve the required concentrations.

\section{ANIMAL STUDIES}

Several in vivo animal studies have been performed evaluating activity of ceftazidime/avibactam. The first study compared activity of ceftazidime/avibactam versus ceftazidime against 27 clinical $P$. aeruginosa isolates in a neutropenic and immunocompetent murine thigh model (37).

Ceftazidime/avibactam MICs ranged from 4$32 \mathrm{mg} / \mathrm{L}$ and dosages which simulated the pharmacodynamics of humans receiving 2000/500mg every 8 hours were utilized. In neutropenic mouse studies, bacterial killing was observed in 16/17 isolates for MICs of $\leq 8 \mathrm{mg} / \mathrm{L}$ and $5 / 8$ for MICs of $16 \mathrm{mg} / \mathrm{L}$ with ceftazidime/avibactam. Efficacy with ceftazidime/avibactam was obtained in all strains in immunocompetent mice at 24 hours with $\geq 0.75 \log$ unit reductions in $13 / 15$ strains compared to $11 / 15$ for ceftazidime alone.

Housman and colleagues evaluated efficacy of ceftazidime/avibactam against $28 \mathrm{P}$. aeruginosa strains in a murine lung model (38). Study dosages were equivalent to human dosing (2000/500mg every 8 hours). For isolates with ceftazidime/avibactam MICs of $\leq 32 \mathrm{mg} / \mathrm{L}$, ceftazidime/avibactam produced $>1 \log _{10}$ CFU reduction in 26/27 strains at 24 hours. Additionally, they observed epithelial lining fluid $f \mathrm{~T}>\mathrm{MIC}$ of $\geq 19 \%$.

Activity of ceftazidime/avibactam has also been studied in animal models against Enterobacteriaceae (39). In a murine thigh model, ceftazidime/avibactam was assessed against 18 strains, 17 resistant to ceftazidime. While ceftazidime monotherapy failed to demonstrate appreciable activity, ceftazidime/avibactam reduced bacterial inoculum in $16 / 18$ strains with no animal succumbing to their infection at experiment duration (24 hours).

\section{CLINICAL DATA (TABLE 2)}




\subsection{Efficacy for the Treatment of Urinary Tract Infections}

In the RECAPTURE 1 and 2 trials ceftazidime/avibactam was evaluated for treatment of complicated urinary tract infections (cUTIs) versus doripenem in a phase III study (40). This was a randomized, prospective, multicenter, doubleblind, double-dummy, comparative study. Eligible patients were 18-90 years diagnosed with acute pyelonephritis or other cUTI necessitating IV therapy. Excluded patients were complete obstruction of any portion of the urinary tract, perinephric or intrarenal abscess, prostatitis, UTI symptoms attributable to another process, urinary diversion, vesicoureteral reflux or creatinine clearance less than $30 \mathrm{~mL} / \mathrm{min}$. Patients meeting criteria were randomized in a $1: 1$ ratio to receive ceftazidime/avibactam 2000/500mg every 8 hours or doripenem $500 \mathrm{mg}$ every 8 hours. The co-primary efficacy endpoints were (1) the proportion of patients with symptomatic resolution of UTI specific symptoms at the day 5 visit; (2) the proportion of patients with both microbiological eradication and symptomatic resolution of all UTI specific symptoms at the test-of-cure (TOC) visit. There were several secondary endpoints including microbiological response at end of IV therapy (EOT) and late follow-up (LFU: 45-52 days post-therapy), per-patient and per-pathogen microbiological response at the TOC and LFU in patients with $\geq 1$ ceftazidime-nonsusceptible or only ceftazidimesusceptible pathogens isolated at baseline, investigator-determined clinical cure, susctained clinical cure at the LFU, and safety. A total of 1033 patients were randomized to receive ceftazidime/avibactam $\quad(n=516)$ or doripenem $(n=517)$. However, the microbiologically modified intention-to-treat (mMITT) population only comprised 810 patients; 393 in the ceftazidime/avibactam group and 417 in the doripenem group. There were no differences between baseline demographics in safety population. Majority $(69.8 \%)$ of patients were female with average age in the early fifties. The majority $(72.0 \%)$ of patients had a primary diagnosis of acute pyelonephritis and only $8.8 \%$ patients with concurrent bloodstream infection. The primary endpoint of symptomatic resolution at day 5, was noted in $276 / 393(70.2 \%)$ and $276 / 417(66.2 \%)$ of patients receiving ceftazidime/avibactam and doripenem, respectively. The additional primary endpoint, patient-assessed symptomatic relief and per-patient favorable microbiological response at TOC, were 280/393 (71.2\%) in ceftazidime/avibactam treated patients and 269/417 $(64.5 \%)$ in doripenem treated patients. Microbiological response rates at EOT and LFU visit in microbiologically evaluable (ME) patients were as follows: $374 / 393(95.2 \%)$ vs 395/417 (94.7\%); $268 / 393 \quad(68.2 \%)$ vs $254 / 417 \quad(60.9 \%)$ for ceftazidime/avibactam and doripenem, respectively. E. coli was the most commonly (73.8\%) isolated uropathogen. Favorable microbiological response rates were comparable between groups irrespective of primary diagnosis or baseline pathogen. A total of 159 ceftazidime-resistant pathogens were isolated (ceftazidime/avibactam: $n=75$; doripenem: $n=84$ ). Despite resistance, microbiological responses at TOC were $62.7 \%$ and $60.7 \%$ in these select patients.

Previous to the RECAPTURE trials, ceftazidime/avibactam was evaluated in a Phase II trial for cUTIs versus imipenem/cilastatin (41). Similarly, patients meeting eligibility criteria were randomized in a 1:1 ratio to receive ceftazidime/avibactam 500/125mg every 8 hours or imipenem/cilastatin 500mg every 6 hours. Primary efficacy endpoint was a favorable microbiological response at TOC visit in ME patients which was assessed 5-9 days after last dosage. One-hundred thirty seven patients were randomized to receive ceftazidime/avibactam $\quad(n=69) \quad$ or imipenem/cilastatin $(n=68)$. However, only 49 ceftazidime/avibactam and 54 imipenem/cilastatin patients completed the study. The primary endpoint, microbiological response at TOC visit, was noted in $19 / 27(70.4 \%)$ and $25 / 35(71.4 \%)$ of patients receiving ceftazidime/avibactam and imipenem/cilastatin, respectively. Microbiological response rates at EOT and LFU visit in ME patients were as follows: $25 / 26(96.2 \%)$ vs $34 / 34(100 \%)$; $15 / 26 \quad(57.7 \%) \quad$ vs $18 / 30 \quad(60.0 \%)$ for ceftazidime/avibactam and imipenem/cilastatin, respectively.

\subsection{Efficacy for the Treatment of Intra- abdominal Infections}

The RECLAIM 1 and 2 trials are the first phase III clinical trials in which ceftazidime/avibactam was evaluated for the treatment of complicated intraabdominal infections (cIAIs) (42). These trials were identical and combined for submission to the US FDA and European Medicines Agency (EMA). They were prospective, randomized, multicenter, doubledummy, double-blind, comparative trials. Eligibility 
included hospitalized patients diagnosed with a cIAI aged 18-90 years (18-65 years in India) who required drainage or surgical intervention within 24 hours of randomization. Patients were specifically excluded if the diagnosis consisted of a traumatic bowel perforation with surgery within 12 hours, perforation of gastroduodenal ulcers with surgical management within 24 hours, an intra-abdominal process unlikely to be infectious, abdominal wall abscess, simple or gangrenous cholecystitis without rupture, infected necrotizing pancreatitis or pancreatic abscess. Included patients were randomized 1:1 to receive ceftazidime/avibactam 2000/500mg every 8 hours plus metronidazole $500 \mathrm{mg}$ every 8 hours or meropenem $1000 \mathrm{mg}$ every 8 hours. The primary end point of noninferiority was clinical cure at TOC (2835 days after randomization) for the following populations: mMITT, modified intention-to-treat (MITT) and clinically evaluable (CE). Several additional secondary endpoints were required by the US FDA and EMA, including (1) clinical response at EOT (within 24 hours after last infusion); and (2) LFU (42-49 days after randomization). There were a total of 1,149 patients enrolled with 1066 randomized to receive therapy (ceftazidime/avibactam plus metronidazole: 532; meropenem: 534). The mMITT, MITT, and CE populations for ceftazidime/avibactam plus metronidazole were 413,520 and 410 and for meropenem were 410, 523 and 416, respectively. Baseline demographics were similar between groups. The primary diagnosis was perforated appendix or periappendicial abscess $(41.9 \%$ ceftazidime/avibactam + metronidazole, $40.7 \%$ meropenem group). Primary efficacy endpoint of clinical cure at TOC was $81.6 \%$ vs $85.1 \%$ for mMITT, $82.5 \%$ vs $84.9 \%$ for MITT and $91.7 \%$ vs $92.5 \%$ for CE populations in the ceftazidime/avibactam plus metronidazole vs. meropenem groups, respectively. Secondary outcomes were as follows: clinical response at EOT was $87.4 \%$ vs $92.4 \%$ for mMITT, $88.3 \%$ vs $92.2 \%$ for MITT and $92.9 \%$ vs $95.2 \%$ with ceftazidime/avibactam plus metronidazole vs meropenem, respectively. Similar results were also observed in clinical response at LFU visit across each population. A total of 111 ceftazidime-resistant pathogens were isolated (ceftazidime/avibactam plus metronidazole: $n=47$; meropenem: $n=64$ ). Regardless of resistance, the clinical response at TOC was $83 \%$ vs $85.9 \%$ for this subset of patients.
A Phase II prospective, randomized, doubleblind cIAIs trial, similar to the Phase III trial, compared ceftazidime/avibactam plus metronidazole versus meropenem (43). Eligible patients were randomized to receive ceftazidime/avibactam 2000/50mg every 8 hours plus intravenous metronidazole $500 \mathrm{mg}$ every 8 hours or meropenem $1000 \mathrm{mg}$ every 8 hours in a 1:1 ratio for a duration of 5-14 days pending clinical response. Primary efficacy endpoint was clinical response at TOC visit in $\mathrm{ME}$ population. Additionally, clinical response was assessed at EOT and TOC visit in CE population, as well as the mMITT population. Twohundred four patients were randomized ( 1 patient did not receive drug) to receive ceftazidime/avibactam plus metronidazole $(n=101)$ or meropenem $(n=102)$. In the ceftazidime/avibactam plus metronidazole and meropenem arms, 68 and 76 ME patients, respectively, were included in primary efficacy endpoint for clinical response. Success rates of 91.2\% (ceftazidime/avibactam plus metronidazole) and $93.4 \%$ (meropenem) were observed. At TOC visit for ME population, overall clinical responses were $91.2 \%$ and $93.4 \%$ for ceftazidime/avibactam plus metronidazole and meropenem patients, respectively.

\subsection{Efficacy for the Treatment of Resistant Organisms}

The REPRISE study was a prospective, open label evaluation to compare ceftazidime/avibactam efficacy to best available therapy for patients with serious infections, specifically cIAIs and cUTIs, with ceftazidime-resistant isolates (44). Patients aged 18-90 with cUTIs or cIAIs were included. Best available therapy was determined by individual investigator following label dosing recommendations. Ceftazidime/avibactam was dosed at 2000/500mg every 8 hours. Primary endpoint was an assessment of clinical response at TOC in the mMITT population. Three hundredthirty three patients were enrolled (ceftazidime/avibactam: 165; best available therapy: 168). However, only 154 and 148 patients were included in the mMITT population. Patient demographics were comparable for the patients treated with either ceftazidime/avibactam or best available therapy for both cUTI and cIAI. The most commonly utilized agents in best available therapy was monotherapy with a carbapenem (97\%) with imipenem/cilastatin or meropenem being the most frequently prescribed. The majority $(92.1 \%)$ of the 
infections were monomicrobial with the most frequently isolated pathogens being $E$. coli and $K$. pneumoniae. However, 11 ceftazidime/avibactam treated patients ( 5 in cUTI group and 6 in cIAI group) and 13 best available therapy treated patients (6 in cUTI group and 7 in cIAI group) had infections with 2 or more isolates identified. Of the 288 isolates identified in this study, only 25 were resistant to ceftazidime/avibactam representing $\quad 91.3 \%$ susceptibility. Overall clinical response rates in mMITT patients were $90.9 \%$ (ceftazidime/avibactam) and 91.2\% (best available therapy). Ten cUTI patients (4 ceftazidime/avibactam treated and 6 best available therapy) in the study had a concomitant bacteremia. However, specific outcomes were not described for these patients.

\subsection{Efficacy for the Treatment of Nosocomial Pneumonia}

A phase III randomized, multicenter, double-blind, double-dummy, parallel-group study to determine the efficacy, safety and tolerability of ceftazidime/avibactam versus meropenem in the treatment of hospital-acquired pneumonia (HAP) including ventilator-associated pneumonia (VAP) has been completed (REPROVE trial) $(45,46)$. Minimal information is available at the time of this writing; however, the manufacturer is expected to provide full results from the study at an upcoming scientific meeting. The limited information released via the Astra Zeneca press releases provided a brief summary of the trial. Adult patients aged 18-90 years were included and randomized to 7 to 14 days of either ceftazidime/avibactam 2000/500mg (infused over 2 hours) given every 8 hours or meropenem $1000 \mathrm{mg}$ (infused over 30 minutes) given every 8 hours. A total of 879 patients were randomized in the study in 23 countries. The primary endpoint for statistical non-inferiority at the test of cure visit on day 21 was achieved with a non-inferiority margin of $12.5 \%$. Additionally, all-cause mortality was comparable between study groups and safety was similar to previous data.

\subsection{Safety}

Overall, ceftazidime/avibactam appears relatively safe from the completed Phase II and III trials for adult and pediatric patients (40-44). However, the majority of additional safety concerns are from the avibactam component given significant historical safety data with ceftazidime alone. Therefore, future usage will enhance the safety profile of this combination product.

\subsubsection{Adverse Reactions}

In the Phase III RECAPTURE trials that evaluated ceftazidime/avibactam versus doripenem for the treatment of cUTIs, drug-related treatment emergent adverse events (AEs) were reported in 185/511 $(36.2 \%)$ ceftazidime/avibactam and 158/509 $(31.0 \%)$ doripenem patients (40). Despite these relatively high numbers, serious adverse events (SAE) leading to discontinuation of the study drugs were substantially lower with only $1.4 \%$ ceftazidime/avibactam patients developing an SAE. The most commonly identified AE consisted of headache which developed in $7.4 \%$ of ceftazidime/avibactam patients and $7.9 \%$ of doripenem treated patients. Gastrointestinal disorders were the second most common $\mathrm{AE}$ reported. Clostridium difficile colitis occurred in 2 of the ceftazidime/avibactam treated patients whereas none of the doripenem treated patients developed this AE. No AEs with an outcome of death were observed in this study. (Table 3 ).

In the Phase II evaluation comparing ceftazidime/avibactam to imipenem/cilastatin for the treatment of cUTIs, AEs were reported in 24/68 (35.3\%) ceftazidime/avibactam and 34/67 (50.7\%) imipenem/cilastatin patients (41). However, only $8.8 \%$ of ceftazidime/avibactam treated patients had an SAE which included acute renal failure, renal impairment, atrial fibrillation, diarrhea, intervertebral disc profusion and accidental overdosage of ceftazidime/avibactam. The accidental overdose patient received four times the amount of drug to be given per study protocol although no AEs were associated with this event (most likely since the dosage received was the approved dose of 2000/500mg). With imipenem/cilastatin, 3.0\% patients developed an SAE (urosepsis:1, blood creatinine increased:1). While there were SAEs, the majority of adverse events were mild. 


\begin{tabular}{|c|c|c|c|c|c|c|c|c|c|c|c|c|c|}
\hline \multirow[t]{2}{*}{ Reference } & \multicolumn{2}{|c|}{$\begin{array}{l}\text { ITT Population } \\
\text { (MITT) }\end{array}$} & \multicolumn{2}{|l|}{ Dose } & \multicolumn{2}{|c|}{ ME success (\%) } & \multirow[t]{2}{*}{ CI } & \multicolumn{2}{|c|}{ CE success (\%) } & \multirow[t]{2}{*}{$\mathrm{CI}$} & \multicolumn{2}{|c|}{ Mortality (n) } & \multirow[t]{2}{*}{ Comments } \\
\hline & CZA & $\mathrm{COM}$ & CZA & $\mathrm{COM}$ & CZA & $\mathrm{COM}$ & & $\mathrm{CZA}$ & $\mathrm{COM}$ & & $\mathrm{CZA}$ & $\mathrm{COM}$ & \\
\hline Ref 41 & $68(46)$ & $67(49)$ & $\begin{array}{l}500 / \\
125 \mathrm{mg} \\
\mathrm{q} 8 \mathrm{~h}\end{array}$ & $\begin{array}{l}\text { IPM } \\
500 \mathrm{mg} \\
\mathrm{q} 6 \mathrm{~h}\end{array}$ & $\begin{array}{l}18 / 27 \\
(66.7)\end{array}$ & $\begin{array}{l}21 / 35 \\
(60.0)\end{array}$ & $\begin{array}{l}-17.4, \\
30.7\end{array}$ & $\begin{array}{l}24 / 28 \\
(85.7)\end{array}$ & $\begin{array}{l}29 / 36 \\
(80.6)\end{array}$ & $\begin{array}{l}-16.3, \\
26.6\end{array}$ & NR & NR & $\begin{array}{l}\text { Phase II: cUTI } \\
\text { CZA vs IPM }\end{array}$ \\
\hline Ref 43* & $101(85)$ & $102(87)$ & $\begin{array}{l}2000 / \\
500 \mathrm{mg} \\
\mathrm{q} 8 \mathrm{~h}\end{array}$ & $\begin{array}{l}\text { MEM } \\
1000 \mathrm{mg} \\
\mathrm{q} 8 \mathrm{~h}\end{array}$ & $\begin{array}{l}62 / 68 \\
(91.2)\end{array}$ & $\begin{array}{l}71 / 76 \\
(93.4)\end{array}$ & $\begin{array}{l}-20.4, \\
12.2\end{array}$ & $\begin{array}{l}80 / 87 \\
(92.0)\end{array}$ & $\begin{array}{l}85 / 90 \\
(94.4)\end{array}$ & $\begin{array}{l}-19.5, \\
10.1\end{array}$ & 3 & 2 & $\begin{array}{l}\text { Phase II: cIAI } \\
\text { CZA+MTZ vs } \\
\text { MEM }\end{array}$ \\
\hline Ref 42 & $532(520)$ & $534(523)$ & $\begin{array}{l}2000 / \\
500 \mathrm{mg} \\
\mathrm{q} 8 \mathrm{~h}\end{array}$ & $\begin{array}{l}\text { MEM } \\
1000 \mathrm{mg} \\
\mathrm{q} 8 \mathrm{~h}\end{array}$ & $\begin{array}{l}276 / 336 \\
(82.1)\end{array}$ & $\begin{array}{l}311 / 356 \\
(87.4)\end{array}$ & NS & $\begin{array}{l}429 / 520 \\
(81.6)\end{array}$ & $\begin{array}{l}444 / 523 \\
(84.9)\end{array}$ & $\begin{array}{l}-6.90, \\
2.10\end{array}$ & $1.3 \%$ & $0.9 \%$ & $\begin{array}{l}\text { RECLAIM 1\&2 } \\
\text { Phase III: cIAI } \\
\text { CZA+MTZ vs } \\
\text { MEM }\end{array}$ \\
\hline Ref 44* & $165(154)$ & $168(148)$ & $\begin{array}{l}2000 / \\
500 \mathrm{mg} \\
\mathrm{q} 8 \mathrm{~h}\end{array}$ & BAT & $\begin{array}{l}126 / 154 \\
(81.8)\end{array}$ & $\begin{array}{l}94 / 148 \\
(63.5)\end{array}$ & NS & $\begin{array}{l}140 / 154 \\
(90.9)\end{array}$ & $\begin{array}{l}135 / 148 \\
(91.2)\end{array}$ & NS & 3 & 4 & $\begin{array}{l}\text { REPRISE } \\
\text { Prospective, open- } \\
\text { label } \\
\text { CZA vs BAT }\end{array}$ \\
\hline Ref 40* & $516(393)$ & $517(417)$ & $\begin{array}{l}2000 / \\
500 \mathrm{mg} \\
\mathrm{q} 8 \mathrm{~h}\end{array}$ & $\begin{array}{l}\text { DOR } \\
500 \mathrm{mg} \\
\mathrm{q} 8 \mathrm{~h}\end{array}$ & $\begin{array}{l}374 / 393 \\
(95.2)\end{array}$ & $\begin{array}{l}395 / 417 \\
(94.7)\end{array}$ & $\begin{array}{l}-2.7 \\
3.55\end{array}$ & $\begin{array}{l}378 / 393 \\
(96.2)\end{array}$ & $\begin{array}{l}407 / 417 \\
(97.6)\end{array}$ & $\begin{array}{l}-4.07, \\
1.02\end{array}$ & 0 & 0 & $\begin{array}{l}\text { RECAPTURE } \\
1 \& 2 \\
\text { Phase III: cUTI }\end{array}$ \\
\hline
\end{tabular}

ITT: intent to treat; MITT: modified intent to treat; CI: confidence interval; CZA: ceftazidime/avibactam; IPM: imipenem/cilastatin; MTZ: metronidazole; MEM: meropenem; BAT: best available therapy dosed according to label recommendations; NR: Not reported; NS: not significant; DOR: doripenem; *data is from the mMITT rather than the MITT 
Safety results from RECLAIM 1 and 2 phase III trials are similar to the aforementioned data with allcause adverse event rates of $45.9 \%$ versus $42.9 \%$ for ceftazidime/avibactam plus metronidazole and meropenem, respectively (42). The most commonly reported events for ceftazidime/avibactam plus metronidazole were gastrointestinal related (diarrhea, nausea, vomiting) ranging from 4.5-7.6\% and fever $4.5 \%$; which was similar to meropenem. Overall, the majority of adverse events were reported as mild to moderate for both study groups with low rates of discontinuation of either arm. The rate of SAE was much lower at $7.9 \%$ for ceftazidime/avibactam plus metronidazole vs $7.6 \%$ in the meropenem group. Although, incidence of SAEs were low, a total of 21 patients died in the study $13 / 529$ (2.5\%) for ceftazidime/avibactam plus metronidazole and 8/529 (1.5\%) for meropenem. The study did not provide any specific information regarding cause or association with study drugs and death. (Table 4).

In the Phase II study for cIAIs, drug-related treatment emergent AEs were reported in 65/101 (64.4\%) ceftazidime/avibactam plus metronidazole and 59/102 (57.8\%) meropenem patients (43). Fortunately, SAEs were infrequent $(8.9 \%$ and $10.8 \%$ ). Three patients expired in the ceftazidime/avibactam plus metronidazole arm (multiorgan failure, sepsis and cardiac arrest) while two died in the meropenem arm (pneumonia and thrombocytopenia). The most commonly recorded treatment-emergent AEs included nausea, vomiting, pyrexia and laboratory abnormalities such as elevated liver enzymes, increased white blood cell count and platelet; however, these were predominantly considered as mild events.

In the REPRISE trial, any patient that received at least one dose of study drug was evaluated for safety endpoints which included 332 of the 333 randomized patients. A total of $31.1 \%$ of ceftazidime/avibactam and $39.3 \%$ best available therapy patients experienced AEs with the most commonly reported being gastrointestinal related (44). Serious adverse events were low at $4.7 \%$ and $6.5 \%$. Discontinuation of study drug due to adverse events occurred in 3 patients: 1 ceftazidime/avibactam, 2 best available therapies with no new safety concerns identified. While 7 patients developed an AE that ultimately led to death (ceftazidime/avibactam: 3 patients; best available therapy: 4 patients), none were attributed to be related to the study drug.

A randomized, placebo-controlled trial was conducted in 18-45 year old healthy males to assess QT prolongation (47). Patients were randomized to receive a single dose of 4 drugs with a minimum 3 day washout period: ceftaroline/avibactam 1500/2000mg, ceftazidime/avibactam 3000/2000mg, moxifloxacin 400mg (positive control) and placebo (negative control). Fifty patients received at least one dose of study drug and included into safety analysis. The largest $90 \%$ CI upper bounds for ceftazidime/avibactam was 5.8 milliseconds, well below the 10 millisecond margin of regulatory concern.

\subsubsection{Drug and Laboratory Reactions}

With minimal Phase III and clinical usage data currently published and nominal time-lapse since approval, few drug interaction data are available. A total of 28 patients were randomized to determine whether there was an interaction between ceftazidime/avibactam and metronidazole over a 4 day period (48). The results observed demonstrated that there was no impact of ceftazidime/avibactam on metronidazole pharmacokinetics or metronidazole on ceftazidime/avibactam pharmacokinetics. In vitro data demonstrates avibactam is a substrate of OAT1 and OAT3 transporters (49).

\begin{tabular}{l}
$\begin{array}{l}\text { Table 3. Frequencies of Adverse Events Occurring in Patients Receiving Ceftazidime/Avibactam or Doripenem } \\
\text { in the RECAPTURE Trials (40) }\end{array}$ \\
\begin{tabular}{lcc}
\hline Adverse Event & Ceftazidime/Avibactam $(\mathrm{n}=511)$ & Doripenem $(\mathrm{n}=509)$ \\
\hline Headache, $\mathrm{n}(\%)$ & $38(7.4)$ & $40(7.9)$ \\
Nausea, $\mathrm{n}(\%)$ & $15(2.9)$ & $10(2.0)$ \\
Diarrhea, $\mathrm{n}(\%)$ & $14(2.7)$ & $6(1.2)$ \\
Constipation, $\mathrm{n}(\%)$ & $11(2.2)$ & $7(1.4)$ \\
Clostridium difficile colitis, $\mathrm{n}(\%)$ & $2(0.4)$ & $0(0)$ \\
\hline *No clinically meaningful trends in laboratory values, electrocardiographic parameters, physical examination, or \\
vital signs were identified
\end{tabular} \\
\hline
\end{tabular}


Probenecid is a known potent OAT inhibitor with potential to decrease elimination of avibactam; therefore, co-administration is not recommended. Additionally, false-positive results for glucose in the urine may occur with administration of ceftazidime. However, to avoid this, enzymatic glucose oxidase reactions can be utilized (9).

\subsubsection{Pregnancy and Breastfeeding}

Like other $\beta$-lactams, ceftazidime/avibactam appears relatively safe in pregnancy though no controlled studies have been performed and is classified as pregnancy category $\mathrm{B}$ due to no evidence of harm with ceftazidime (mice and rats) and no teratogenic effects for avibactam (rats or rabbits) (9). No data exist regarding excretion of avibactam in human milk but ceftazidime has low concentrations. In case of nursing mothers, benefit should outweigh the risk with incomplete available data.

\section{POST-MARKETING DATA / CASE SERIES / CASE REPORT}

Since FDA approval, minimal post-marketing data exist regarding the utility of ceftazidime/avibactam. Three case series were recently presented at the 2016 American Society for Microbiology Microbe meeting (1 of which is now published), as well as 1 published case report, which are briefly described below.

The largest case series described the clinical outcomes associated with ceftazidime/avibactam in patients with CRE infections (50). The most prevalent infection types were pneumonia (32\%) and bacteremia (27\%). A median duration of therapy of 14 days yielded clinical success in 59\% (22/37) of the patients. Of these 22 successfully treated patients, $5(23 \%)$ had a relapse of CRE infection at the same or contiguous site. It is important to note, however, that combination therapy, mostly with aminoglycosides, was provided for approximately a third of these patients. However, clinical success was comparable between those treated with ceftazidime/avibactam as monotherapy or combination therapy ( $58 \%$ vs. $64 \%$ ).

The second largest case series evaluated ceftazidime/avibactam against gram-negative bacilli (51). All 15 patients included in this analysis received ceftazidime/avibactam for compassionate use after previous receipt of standard therapy. The median duration of therapy for ceftazidime/avibactam was 24 days (range 3-50 days), which provided clinical success in 5/11 (45\%) patients (4 had indeterminate outcomes). The most frequently identified organism was $K$. pneumonia (12/15 isolates). Eighty percent of the isolates were susceptible to ceftazidime/avibactam with microbiological success observed in 13/15 (87\%) patients. Despite the microbiological success, delayed usage and severity of illness attributed to minimal clinical success and high mortality $(67 \%)$.

The smallest case series to date included 9 patients that received ceftazidime/avibactam for CRE infections (52). Of these cases, K. pneumoniae was the organism isolated in 8 of 9 patients. The most frequent infection type was pneumonia (55.6\%) followed by urinary tract infection (22.2\%). Microbiological cure and 30-day mortality were documented in 55\% and 22\%, respectively. Clinical success was higher in this case series at 55\% (5 of 9 patients); however, fewer patients had bacteremia than in the previously discussed case series.

Lastly, a single report of ceftazidime/avibactam was published with minimal patient information for an off-label indication of a 62 year old female with a complicated past medical history presenting with vomiting and fever two weeks after a pylorus preserving pancreaticoduodenectomy (20). Admission blood cultures were positive for Citrobacter freundii and a carbapenem-resistant $K$. pneumoniae. Initial treatment was gentamicin and cefepime, with subsequent therapy changed to colistin, meropenem and tigecycline. As the patient continued to worsen from nephrotoxic effects of the antibiotics in conjunction with sepsis, ceftazidime/avibactam was added on hospital day 21 through compassionate use for enhanced coverage of the CRE. Cultures were positive for the CRE on days $1,8,9,20$ and 22, with all further cultures having no growth. Ceftazidime/avibactam MICs were $4 / 4$ and $32 / 4 \mathrm{mg} / \mathrm{L}$ for blood isolates and $8 / 4 \mathrm{mg} / \mathrm{L}$ for isolates from both liver and sputum. The patient eventually continued treatment with intravenous trimethoprim/sulfamethoxazole and colistin for ongoing hepatic abscesses. The patient case report was published while the patient was still receiving the above regimen with no additional outcome information provided.

\section{FUTURE DEVELOPMENT}

(clinicaltrials.gov)

According to clinicaltrials.gov, several studies are in various stages of development to further assess the activity of ceftazidime/avibactam. Currently, 3 
studies are in the recruitment phase on research. A phase III, multicenter, double-blind, double-dummy trial evaluating ceftazidime/avibactam versus meropenem for the treatment of nosocomial pneumonia among hospitalized adults with an enrollment of 969 patients completed earlier in 2016 with results yet to be published. Additionally, two pediatric studies are currently enrolling patients. The first is an evaluation of the safety, efficacy and pharmacokinetics of ceftazidime/avibactam versus cefepime for the treatment of complicated UTIs in children 3 months of age to 18 years. Approximately 100 patients are estimated to enroll with a projected completion date of March 2017. A similar study, in children 3 months of age to 18 years, is currently enrolling patients for the safety and efficacy of ceftazidime/avibactam plus metronidazole versus meropenem for the treatment of complicated IAIs. Similarly, approximately 100 patients will be enrolled with an expected completion date of April 2017. Lastly, a study evaluating the pharmacokinetics in cystic fibrosis patients with an enrollment of 12 patients was recently completed but results are not yet available.

\section{CONCLUDING REMARKS}

Ceftazidime/avibactam has demonstrated comparable efficacy to imipenem/cilistatin and doripenem in cUTIs and with metronidazole vs meropenem in cIAIs, as well as compared to meropenem for HAP, including VAP. This agent increases the current antimicrobial armamentarium for MDR gram-negative pathogens, particularly Enterobacteriaceae, with similar adverse events as other $\beta$-lactams. Of primary importance is the expanded activity against ESBLs and CREs which will likely be this agent's primary role in therapy. Additionally, this agent may retain activity against some Pseudomonas aeruginosa, but other newer agents may be better suited for this organism. Despite the enhanced activity, with limited agents for MDR gram-negatives, clinicians should conserve this agent for use against pathogens where other less broad agents still remain an option. Furthermore, it will be important to continue to monitor outcomes with indications other than the approved cUTIs or cIAIs, and HAP/VAP (Europe) to determine whether this agent will be a viable alternative in those infections.

\section{POST SUBMISSION NOTE}

Future Concerns: Albeit ceftazidime/avibactam is a welcome addition for the treatment of multidrugresistant gram-negatives, questions exist of the long standing utility of this agent (53). While ceftazidime has always been linked to emergence of resistance, the potency of avibactam for inhibition of carbapenemases (KPCs) made the combination attractive, particularly for CRE isolates. However, with avibactam's limited activity against metallo- $\beta$ lactamases, the broad effectiveness of this combination agent is reduced. In regards to activity, clinical trials in complicated UTIs and IAIs demonstrated non-inferiority; however, there were several caveats. The first being in cIAIs patients with a $\mathrm{CrCl}<50 \mathrm{~mL} / \mathrm{min}$, where clinical cure was $45.2 \%$ for ceftazidime/avibactam plus metronidazole vs $74.3 \%$ meropenem in the mMITT group and $72 \%$ vs $88 \%$ in the CE group at TOC (42). The second concern being no CRE isolates were included in any of the clinical trials. However, recent case series have specifically reviewed patients with CRE infections treated with ceftazidime/avibactam (5052). While efficacy was obtained in many of the treated patients, the overall success was worrisome (range 45-59\%), relapse rates were higher than desired even with combination therapy (up to 23\%), and there was development of resistance to ceftazidime/avibactam (MIC $\geq 16 \mathrm{mg} / \mathrm{L}$ ) following relatively short courses of therapy (10-19 days) (5052). Although, these case series represent a small sample size, it does give rise to the fear of having only this agent for treatment of CRE infections. It is crucial for additional clinical trials evaluating the efficacy and prevention of resistance for CRE organisms to be conducted. However, for now, while this old agent, ceftazidime, has been "taught a new trick" with the addition of avibactam, especially for ESBL isolates - on the other hand CRE infections may prove to be this combination's "short leash" with eventual restriction in long term utility.

\section{ACKNOWLEDGEMENTS}

This research was performed without external funding. KEB has received research grant support from Allergan, Plc. JKO has nothing to disclose. RLA has received research grant support from Cubist Pharmaceuticals, Inc., Cerexa, Inc. and Theravance Biopharma.

\section{REFERENCES}


1. Antibiotic resistance threats in the United States, 2013. Atlanta, GA: Centers for Disease Control and Prevention. http://cdc.gov/drugresistance/threatreport-2013/ (accessed 2015 Aug 6).

2. Jacoby GA, Munoz-Price LS. The new betalactamases. N Engl J Med. 2010;352:380-391. doi: 10.1056/NEJMra041359

3. Spanik S, Krupova I, Trupl J, et al. Bacteremia due to multiresistant gram-negative bacilli in neutropenic cancer patients: a case-controlled study. J Infect Chemother. 1999;5:180-184.

4. Coleman K. Diazabicyclooctanes (DBOs): a potent new class of non- $\beta$-lactam- $\beta$ -

lactamase inhibitors. Curr Opin Microbiol. 2011;14:550-555.

5. Draft guidance for industry: antibacterial therapies jfor patients with unmet medical need for the treatment of serious bacterial dieases. Rockville, MD: Food and Drug Administration, 2013. http://www.fda.gov/downloads/drugs/guidanceCom plianceRegulatoryInformation/guidances/uCM35918 4.pdf (accessed 2015 Aug 3).

6. Caprile KA. The cephalosporin antimicrobial agents: a comprehensive review. J Vet Pharmacol Ther. 1988;11:1-32.

7. Marsh TD. The cephalosporin antibiotic agents-III. Third-generation cephalosporins. Infect Control. 1985;6:78-83.

8. Hayes MV, Orr DC. Mode of action of ceftazidime: affinity for the penicillin-binding proteins of Escherichia coli K12, Pseudomonas aeruginosa and Staphylococcus aureus. J Antimicrob Chemother. 1983;12:119-126.

9. Product information. Avycaz (ceftazidimeavibactam) injection. Cincinnati, OH: Forest Pharmaceuticals, Inc., February 2015.

10. Castanheira M, Mills JC, Costello SE, Jones RN, Sader HS. Ceftazidime-avibactam activity tested against Enterobacteriaceae isolates from U.S. hospitals (2011 to 2013) and characterization of $\beta$ lactamase-producing strains. Antimicrob Agents Chemother. 2015;59:3509-3517. doi: 10.1128/AAC.00163-15

11. Aktas Z, Kayacan C, Oncul O. In vitro activity of avibactam (NXL104) in combination with $\beta$-lactams against gram-negative bacteria, including OXA-48 $\beta$ lactamase-producing Klebsiella pneumoniae. Int $\mathrm{J}$ Antimicrob Agents. 2012;39:86-89. doi: 10.1016/j.ijantimicag.2011.09.012

12. Sader HS, Castanheira M, Mendes RE, Flamm RK, Farrell DJ, Jones RN. Ceftazidime-avibactam activity against multidrug-resistant Pseudomonas aeruginosa isolated in U.S. medical centers in 2012 and 2013. Antimicrob Agents Chemother. 2015;59:3656-3659. doi: 10.1128/AAC.05024-14

13. Flamm RK, Farrell DJ, Sader HS, Jones RN. Ceftazidime/avibactam activity tested against gram- negative bacteria isolated from bloodstream, pneumonia, intra-abdominal and urinary tract infections in US medical centres (2012). J Antimicrob Chemother. 2014;69:1589-1598. doi: 10.1093/jac/dku025

14. Dubreuil LJ, Mahieux S, Neut C, Miossec C, Pace J. Anti-anaerobic activity of a new $\beta$-lactamase inhibitor NXL104 in combination with $\beta$-lactams and metronidazole. Int $\mathrm{J}$ Antimicrob Agents. 2012;39:500-504. doi: 10.1016/j.jiantimicag.2012.02.013

15. O'Callaghan $\mathrm{CH}$, Acred P, Harper PB, Ryan DM, Kirby SM, Harding SM. GR 20263, a new broadspectrum cephalosporin with anti-pseudomonal activity. Antimicrob Agents Chemother. 1980;17:876-883.

16. European Centre for Disease Prevention and Control (ECDC). Antimicrobial resistance surveillance in Europe 2014. Annual Report of the European Antimicrobial Resistance Surveillance Network (EARS-Net). Stockholm: ECDC;2015. Available from

http://ecdc.europa.eu/en/publications/Publications/an timicrobial-resistance-europe-2014.pdf

17. European Centre for Disease Prevention and Control (ECDC). Antimicrobial resistance interactive database (EARS-Net). Stockholm: ECDC. [Accessed 19 Nov 2015]. Available from http://ecdc.europa.eu/en/healthtopics/antimicrobial_r esistance/database/Pages/database.aspx

18. Sader HS, Castanheira M, Flamm RK, Farrell DJ, Jones RN. Antimicrobial activity of ceftazidimeavibactam against gram-negative organisms collected from U.S. medical

centers in 2012. Antimicrob Agents Chemother. 2014;58:1684-92.

19. Livermore DM, Warner M, Jamrozy D, et al. In vitro selection of ceftazidime-avibactam resistance in Enterobacteriaceae with KPC-3 carbapenemase. Antimicrob Agents Chemother. 2015;59:5324-5330. doi: 10.1128/AAC.00678-15

20. Humphries RM, Yang S, Hemarajata P, et al. First report of ceftazidime-avibactam resistance in a KPC3 expressing Klebsiella pneumoniae. Antimicrob Agents Chemother. 2015. [Epub ahead of print] doi:10.1128/AAC.01165-15

21. Shields RK, Clancy CJ, Hao B, et al. Effects of Klebsiella pneumoniae carbapenemase subtypes, extended-spectrum $\beta$-lactamases, and porin mutations on the in vitro activity of ceftazidimeavibactam against carbapenem-resistant $\mathrm{K}$. pneumoniae. Antimicrob Agents Chemother. 2015;59:5793-5797. doi: 10.1128/AAC.00548-15

22. Lahiri SD, Walkup GK, Whiteaker JD, et al. Selection and molecular characterization of ceftazidime-avibactam-resistant mutants in Pseudomonas aeruginosa strains containing 
derepressed AmpC. J Antimicrob Chemother. 2015;70:1650-1658. doi: 10.1093/jac/dkv004

23. Miossec C, Claudon M, Levasseur P, Black MT. The $\beta$-lactamase inhibitor avibactam (NXL104) does not induce ampC $\beta$-lactamase in Enterobacter cloacae. 2013;6:235-240.

24. Porres-Osante N, Dupont H, Torres C, et al. Avibactam activity against extended-spectrum AmpC $\beta$-lactamases. J Antimicrob Chemother. 2014;69:1715-1716. doi: 10.1093/jac/dku002

25. Livermore DM, Mushtaq S, Warner $\mathrm{M}$, et al. Activities of NXL104 combinations with ceftazidime and aztreonam against carbapenemase producing Enterobacteriaceae. Antimicrob Agents Chemother. 2011;55:390-394. doi: 10.1128/AAC.00756-10

26. Livermore DM. Of Pseudomonas, porins, pumps and carbapenems. J Antimicrob Chemother. 2011;47:247-250.

27. Winkler ML, Papp-Wallace KM, Hujer AM, et al. Unexpected challenges in treating multidrug-resistant gram-negative bacteria: resistance to ceftazidimeavibactam in archived isolates of Pseudomonas aeruginosa. Antimicrob Agents Chemother. 2015;59:1020-1029. doi: 10.1128/AAC.04238-14

28. Merdjan H, Rangaraju M, Tarral A. Safety and_pharmacokinetics_of single and multiple ascending doses of avibactam alone and in combination with ceftazidime in healthy male volunteers: results of two randomized, placebocontrolled studies. Clin Drug Investig. 2015;35:307317. doi: 10.1007/s40261-015-0283-9

29. Tarral A, Merdjan H. Effect of age and sex on the pharmacokinetics and safety of avibactam in healthy volunteers. Clin Ther. 2015;37:877-886. doi: 10.1016/j.clinthera.2015.01.009

30. Tominaga N, Edeki T, Li J, Learoyd M, Bouw MR, Das S. Phase I study assessing the safety, tolerability, and pharmacokinetics of avibactam and ceftazidimeavibactam in healthy Japanese volunteers. J Infect Chemother. 2015;21:551-518. doi: 10.1016/j.jiac.2015.04.006

31. Merdjan H, Tarral A, Haazen W, Evene E, Robertson M, Sable C. Pharmacokinetics and tolerability of NXL104 in normal subjects and patients with varying degrees of renal insufficiency [abstract P1598]. 20 $0^{\text {th }}$ European Congress of Clinical Microbiology and Infectious Diseases. Vienna, Austria. April 10-13, 2010.

32. Bradley J, Armstrong R, Das $\mathrm{S}$ et al. Single-dose pharmacokinetics (PK) of ceftazidime-avibactam (CAZ-AVI) in hospitalized pediatric patients [abstract G-1205]. Interscience Conference on Antimicrobial Agents and Chemotherapy Meeting/International Congress of Chemotherapy and Infection. San Diego, CA. September 17-21, 2015.
33. Richards DM, Brogden RN. Ceftazidime. A review of its antibacterial activity, pharmacokinetic properties and therapeutic use. Drugs. 1985;29:10561.

34. Drusano GL. Prevention of resistance: a goal for dose selection for antimicrobial agents. Clin Infect Dis. 2003;36(Suppl 1):S42-S50.

35. Noel AR, Tomaselli SG, Nicholls DL, Bowker KE, Williams G, MacGowan AP. The pharmacodynamics of avibactam (NXL104) in combination with either ceftaroline or ceftazidime against $\beta$-lactamase producing Enterobacteriaceae [abstract A-631]. 52nd Annual Interscience Conference on Antimicrobial Agents and Chemotherapy. San Francisco, CA. September 9-12, 2012.

36. Bowker KE, Noel AR, MacGowan AP. Pharmacodynamics of NXL104 plus either ceftaroline or ceftazidime against an AmpC producing Enterobacter spp. [abstract A2-556]. 51st Annual Interscience Conference on Antimicrobial Agents and Chemotherapy. Chicago, IL. September 17-20, 2011.

37. Crandon JL, Schuck VJ, Banevicius MA, et al. Comparative in vitro and in vivo efficacies of human simulated doses of ceftazidime and ceftazidimeavibactam against Pseudomonas_aeruginosa. Antimicrob Agents Chemother. 2012;56:6137-6146. doi: 10.1128/AAC.00851-12

38. Housman ST, Crandon JL, Nichols WW, Nicolau DP. Efficacies of ceftazidime-avibactam and ceftazidime against Pseudomonas aeruginosa in a murine lung infection model. Antimicrob Agents Chemother. 2014;58:1365-1371. doi: 10.1128/AAC.02161-13

39. MacVane SH, Crandon JL, Nichols WW, Nicolau DP. In vivo efficacy of humanized exposures of Ceftazidime-Avibactam in comparison with Ceftazidime against contemporary Enterobacteriaceae isolates. Antimicrob Agents Chemother. 2014;58:6913-6919. doi: 10.1128/AAC.03267-14

40. Wagenlehner FM, Sobel JD, Newell $P$, et al. Ceftazidime-avibactam Versus Doripenem for the Treatment of Complicated Urinary Tract Infections, Including Acute Pyelonephritis: RECAPTURE, a Phase 3 Randomized Trial Program. Clin Infect Dis. 2016 Sep 15;63(6):754-62. doi: 10.1093/cid/ciw378.

41. Vazquez JA, González Patzán LD, et al. Efficacy and safety of ceftazidime-avibactam versus imipenemcilastatin in the treatment of complicated urinary tract infections, including acute pyelonephritis, in hospitalized adults: results of a prospective, investigator-blinded, randomized study. Curr Med Res Opin. 2012;28:1921-1931. doi: 10.1185/03007995.2012.748653

42. Mazuski JE, Gasink L, Armstrong J, et al. Efficacy and safety of ceftazidime-avibactam plus 
metronidazole versus meropenem in the treatment of complicated intra-abdominal infection: results from a randomized, controlled, double-blind, phase 3 program. Clin Infect Dis. 2016;62:1380-1389. doi: 10.1093/cid/ciw133.

43. Lucasti C, Popescu I, Ramesh MK, Lipka J, Sable C. Comparative study of the efficacy and safety of ceftazidime/avibactam plus metronidazole versus meropenem in the treatment of complicated intraabdominal infections in hospitalized adults: results of a randomized, double-blind, Phase II trial. J Antimicrob Chemother. 2013;68:1183-1192. doi: 10.1093/jac/dks523

44. Carmeli Y, Armstrong J, Laud P, et al. Ceftazidimeavibactam or best available therapy in patients with ceftazidime-resistant Enterobacteriaceae and Pseudomonas aeruginosa complicated urinary tract infections or complicated intra-abdominal infections (REPRISE): a randomized, pathogen-directed, phase 3 study. Lancet Infect Dis. 2016;16:661-673. doi: http://dx.doi.org/10.1016/S1473-3099(16)30004-4

45. AstraZeneca press release. AstraZeneca's antibiotic Zavicefta met primary endpoints in Phase III trial for treatment of hospital-acquired pneumonia. https://www.astrazeneca.com/media-centre/pressreleases/2016/AstraZenecas-antibiotic-Zaviceftamet-primary-endpoints-in-Phase-III-trial-fortreatment-of-hospital-acquired-pneumonia21072016.html. July 21, 2016. Accessed: August 12, 2016.

46. European Medicines Agency. European public assessment report (EPAR): Zavicefta (ceftazidime/avibactam).

http://www.ema.europa.eu/docs/en_GB/document_li brary/EPAR_-

Summary_for_the_public/human/004027/WC5002 10237.pdf. June 24, 2106. Accessed: August 12, 2016.
47. Das S, Armstrong J, Mathews D, Li J, Edeki T. Randomized, placebo-controlled study to assess the impact on QT/QTc interval of supratherapeutic doses of ceftazidime-avibactam or ceftaroline fosamilavibactam. J Clin Pharmacol. 2014;54:331-340. doi: 10.1002/jcph.199

48. Das $\mathrm{S}, \mathrm{Li} \mathrm{J}$, Armstrong J, Learoyd M, Edeki T. Randomized pharmacokinetic and drug-drug interaction studies of ceftazidime, avibactam, and metronidazole in healthy subjects. Pharmacol Res Perspect. 2015;3:e00172.

49. Vishwanathan $\mathrm{K}^{1}$, Mair S, Gupta A, et al. Assessment of the mass balance recovery and metabolite profile of avibactam in humans and in vitro drug-drug interaction potential. Drug Metab Dispos. 2014;42:932-942. doi: 10.1124/dmd.113.055335

50. Shields RK, Potoski BA, Haidar G, et al. Clinical outcomes, drug toxicity and emergence of ceftazidime-avibactam resistance among patients treated for carbapenem-resistant Enterobacteriaceae infections. Clin Infect Dis. 2016 Sep 13. pii: ciw636. [Epub ahead of print]

51. Gallagher JC, McKinnell J, CAZAVI Compassionate Use Research Group. Compassionate use of ceftazidime/avibactam (Cazavi) for carbapenemresistant $(\mathrm{Cr})$ gram-negative rod (Gnr) infections, abstr 533. Abstr ASM Microbe. Amer Soc Microbiol. Boston, MA, USA, June 20, 2016.

52. King M, Gallagher J. Clinical outcomes with ceftazidime-avibactam in patients with carbapenemresistant Enterobacteriaceae (Cre) infections, abstr 428. Abstr ASM Microbe. Amer Soc Microbiol. Boston, MA, USA, June 17, 2016.

53. Spellberg B, Bonomo RA. Ceftazidime-avibactam and carbapenem-resistant Enterobacteriaceae: "We're gonna need a bigger boat". Clin Infect Dis. 2016. [Epub ahead of print]. DOI: 10.1093/cid/ciw639 


\begin{tabular}{lcc}
\hline $\begin{array}{l}\text { Table 4. Frequencies of Adverse Events Occurring up to LFU visit (6-7 weeks from randomization) in Patients } \\
\text { Receiving Ceftazidime/Avibactam plus Metronidazole or Meropenem (42) }\end{array}$ \\
$\begin{array}{l}\text { Ceftazidime/Avibactam plus } \\
\text { Metronidazole (n=529) }\end{array}$ & Meropenem (n=529) \\
Adverse Event & $2.1 \%$ & $1.7 \%$ \\
Anemia & $2.8 \%$ & $1.7 \%$ \\
Headache & $2.8 \%$ & $4.5 \%$ \\
Hypertension & $2.3 \%$ & $2.3 \%$ \\
Hypotension & $1.9 \%$ & $2.1 \%$ \\
Phlebitis & $2.1 \%$ & $2.5 \%$ \\
Cough & $7.6 \%$ & $3.4 \%$ \\
Diarrhea & $6.8 \%$ & $4.5 \%$ \\
Nausea & $4.5 \%$ & $1.9 \%$ \\
Vomiting & $1.9 \%$ & $2.1 \%$ \\
Abdominal distention & $1.5 \%$ & $3.8 \%$ \\
Constipation & $4.5 \%$ & $4.5 \%$ \\
Pyrexia & $1.9 \%$ & $2.3 \%$ \\
Asthenia & $2.1 \%$ & $1.5 \%$ \\
Liver disorder & $4.3 \%$ & $3.0 \%$ \\
Hypersensitivity/anaphylaxis & $3.0 \%$ & $2.8 \%$ \\
Hematological disorder & $2.3 \%$ & $0.6 \%$ \\
Renal disorder & &
\end{tabular}

